\title{
Graphene-based Flexible and Stretchable Bioelectronics in Health Care Systems
}

\begin{abstract}
The objective of this short review is to summarize the recent applications of graphene in the field of flexible and stretchable bioelectronics. The review highlights the current developments in graphene and graphene hybrid based bioelectronics and their properties (in terms of stretchability and conductivity), challenges and future perspectives.
\end{abstract}

Volume 3 Issue 2 - 2016

\author{
Vinod Kumar, Gaurav Khandelwal \\ 'Ben-Gurion University of the Negev, Israel \\ ${ }^{2}$ University of Rajasthan, India
}

Correspondence: Vinod Kumar, Ben-Gurion University of the Negev, Beer-Sheva 84105, Israel, Email mail2vinod@gmail.com

Received: September 26, 2016 | Published: October 06, 2016

\section{Introduction}

Nanomaterials are playing a vital role to meet increasing demand of bioelectronics devices in health care system. In this context, different kind of nanomaterials has been examined. In recent years emergence of 2-D nanomaterials like graphene have been largely explored in various technological application including bioelectronics. Current mini review provides a meaningful insight towards potential application of graphene in developing efficient flexible and stretchable bioelectronics devices in healthcare system.

Efficient bimolecular detection is very important for biomedical, environmental, as well for security purposes. This has been become possible by means of efficient analytical devices or by biosensors. In recent years, biosensors research has been largely explored in the area of biomedicine, environmental screening in safety. Although the existing biosensing technology is contributing well in the concerned area, even though there is large scope to improve their performance for better outcomes. The application of biosensors in general public health care system needs to be largely addressed. Since the discovery of field effect transistor in the 1920s, there is tremendous efforts have been made in electronic industries to develop devices with high speed and large capacity (such as microprocessors and random access memories). Recent growth of personalized and mobile electronics has expanded and tuned the research domains in electronics from performance-oriented to more in human health care topics. ${ }^{1}$ Thus, further expansion of electronics in developing flexible and stretchable biomimetic systems have been initiated. ${ }^{2}$ As results, in short period of time, the market size of health care gadgets is significantly increased with further expectations of more improved medical devices. ${ }^{3}$

The high expectations of the flexible electronics in healthcare system are due to their high flexibility, inexpensive substrate material and low-cost of manufacturing etc. However, the stretchability was the only limitations with flexible devices, ${ }^{4}$ which have been overcome by incorporating inorganic and organic nanomaterials. ${ }^{5-9}$ Thus flexible and stretchable devices have become a powerful alternatives to bulky health-monitoring devices. Nanomaterials enabled flexible and stretchable bioelectronics devices have attracted significant interest in healthcare applications. It is due to the several unique features of nanomaterials including medical multifunctionality, mechanical deformability, and other excellent properties etc. ${ }^{9}$ Nanomaterials with superior properties for e.g. large surface areas, high mechanical strength, and high electronic conductivity, are highly compatible for developing more efficient and large-scale flexible and stretchable electronic devices. ${ }^{10,11}$ Till date, application of nanomaterials as stretchable interconnects or conductors is reported, while stretchable and flexible active device components based on nanomaterials. ${ }^{12,13}$ remains as an emerging area of research. Continuous efforts are being made to utilize novel properties of nanomaterials in health care bioelectronics. Several nanomaterials have been explored in developing efficient diagnostic systems ${ }^{14}$ and other are under exploration. The currentshort note provides a summary of application of graphene, a 2-D carbon nanomaterial, in flexible and stretchable electronic devices.

\section{Graphene-Based Stretchable and Flexible Devices}

Polydimethylsiloxane (PDMS), an elastomer has been widely used in the substrate for incorporation of nanomaterials for developing flexible and stretchable bioelectronic devices. A composite of nanomaterials and PDMS can also be made to enhance the stretchability and flexibility of the devices.

Graphene, a 2-D layered material (an allotrope of carbon), possesses unique features, have made a tremendous impact in the field of nanoscience and technology. Discoverer of graphene received Nobel Prize in Physics in the year 2010. Since its discovery by mechanical exfoliation of graphite, ${ }^{15,16}$ graphene has become a material of central interest due to its unique 2-D layered structure, and its unique physic-chemical properties, for e.g. excellent electron mobility, high chemical and thermal stability, ultra-high strength, large specific 2-D surface area, and low contact resistance with organic electronic devices. ${ }^{17}$ Innumerable applications of graphene have been demonstrated, such as in electrochemical sensors and biosensors, polymer composites, field-effect transistors and organic electronic devices, and energy conversion and storage devices. ${ }^{18}$ Numerous methods have been established for e.g. epitaxial growth on $\mathrm{SiC}^{19,20}$ and metal surfaces, ${ }^{21}$ reduction from graphite oxide, ${ }^{22}$ liquid-phase exfoliation ${ }^{23}$ and Chemical Vapor Deposition (CVD). ${ }^{24}$ However, CVD is comparatively simple and feasible method for large scale production high-quality graphene. Synthesized graphene can be transferred onto random substrates with the help of range of polymers like PDMS [25], poly(methyl methacrylate) (PMMA) [26] or thermal release tape [27]. The transferred graphene films displayed a high quality and sheet resistance of ca. $30 \Omega$ sq- 1 at ca. $90 \%$ transmittance was realized for $\mathrm{p}$-doped 4-layered graphene. 


\section{Fabrication of Graphene Devices and Properties}

Comparatively high transparency and good electrical conductivity of graphene make it a promising candidate as transparent electrodes. ${ }^{28}$ Large-scale, patterned synthesis of high-quality graphene by CVD method and its effective transfer onto different substrates is reported. ${ }^{25}$ The graphene film was transferred to biaxially prestrained PDMS (ca. $12 \%$ strain) to enhance electromechanical stability. Both longitudinal and transverse resistance remained stable within $11 \%$ tensile strain and increased by one order of magnitude at ca. $25 \%$ strain after releasing the PDMS. Alternatively, to make stretchable graphene film, one need to grow the graphene on wavy $\mathrm{Cu}$ foil (instead of the planar one), followed by drop-casting PDMS and etching the $\mathrm{Cu}$ foil. ${ }^{29}$ The resulting graphene/PDMS showed transmittance between $50 \%$ and $60 \%$. Coating of another layer of PVA on top of the graphene largely suppresses the creation of cracks during stretching and thus enhanced the stretchability. The resistance of PVA coated graphene/ PDMS is increased by $\sim 2$ times under tensile strain of $40 \%$. Selforganized, crumpled hierarchical structures can be formed by sequentially releasing the biaxially prestrained VHB 4905 substrate with a graphene film on top along the two prestrain directions. ${ }^{30}$ The crumpled structure can be "unfolded when stretching the substrate back in both directions. The crumpled graphene conductor can accommodate an extreme strain of $450 \%$ along the direction with the higher prestrain, with unequal prestrains of $10 \%$ and $500 \%$. The crumpled make graphene super hydrophobic. And the wettability and optical transmittance of the graphene can be tuned by reversible crumpling-unfolding process. Further, 3-D macroscopic graphene foams (GFs) were synthesized by a template-directed CVD using nickel foam as a template. ${ }^{31}$ The well-interconnected 3D conducting network in GFs, the GF/PDMS composite with low graphene loading of ca. $0.5 \mathrm{wt} \%$ have shown good conductivity of ca. $10 \mathrm{~S} \mathrm{~cm}^{-1}$ and high fracture stain of ca. $95 \%$. After being treated by five cycles of stretching, the resistance increased ca. $30 \%$ under 50\% tensile strains.

\section{Fabrication of Devices using Graphene- Hybrid Materials and their Properties}

Doping of foreign atoms in graphene improves its electrical properties. Several methods of doping have been introducing, to enhance the conductivity of graphene, such as chemical doping, ${ }^{32}$ introducing $\mathrm{CNTs}^{33}$ and metal nanostructures (e.g. depositing on top of graphene or mixing with graphene) $)^{34}$ etc. Moreover, Graphene$\mathrm{Ag}$ hybrid fibers were synthesized by the wet-spinning process and subsequent chemical reduction, and they exhibited enhanced conductivity and current capacity. ${ }^{35}$ Ag-doped graphene fibers transferred onto PDMS pre-strained by $150 \%$, the conductivity was maintained for strains within $150 \%$. Conductivity of graphene has been further enhanced by spin-coating a thin layer of AgNW network on its top, where the conducting pathways of the graphene and AgNWs function complementary to each other, and overcome to charge scattering by defects in grapheme ${ }^{36}$ and as results, a low resistance of $33 \Omega \mathrm{sq}^{-1}$ at a high transmittance of $94 \%$ was obtained. Graphene-AgNW on PDMS have shown a large strain tolerance of $100 \%$ with negligible resistance degradation, in addition, the hybrid structure showed a much higher breakdown electric field and better stability against thermal oxidation compared with pure AgNWs. Apart from the graphene, other 2-D layered materials like $\mathrm{MoS}_{2}, \mathrm{WS}_{2}$, and $\mathrm{VS}_{2}$ are under evaluation for their further application in flexible and stretchable devices.

\section{Challenges}

For efficient application of graphene in developing flexible and stretchable bioelectronics systems, some specific challenges need to be thoroughly addressed:

a. Facile integration technology of graphene in bio-electronic devices.

b. Through interaction study of graphene with supporting substrate.

c. Through characterization of graphene-based bioelectronics devices in extreme conditions.

\section{Conclusion}

Increasing demand for flexible and stretchable electronic devices in health care system accelerated the development of more efficient bioelectronics devices. Incorporation of nanomaterials for developing novel bioelectronics devices provides immense opportunities for advanced diagnostics and drug delivery. 2-D layered materials like graphene along with other upcoming materials have huge potential for significantly improving the performance of existing devices. The Huge potential of nanomaterials based flexible and stretchable bioelectronics needs to be thoroughly investigated especially for health biosystem. Nanomaterials enabled flexible and stretchable bioelectronics devices may lead to significant expansion and application of these devices in healthcare system.

\section{Acknowledgements}

Dr. Vinod Kumar is a postdoctoral scientist at Ben-Gurion University of the Negev, Israel. He received his $\mathrm{PhD}$ in Nanobiotechnology from Banaras Hindu University, India in 2014. His current research interest focuses on design and development of nanomaterial based high efficient flexible and stretchable point of care devices for early and accurate screening of disease biomarkers.

Mr. Gaurav Khandelwal is a Project Associate at Indian Institute of Technology Delhi, India. He completed his M. Tech in Nanotechnology from Center for Converging Technologies, University of Rajasthan, India. His current research interest includes Nanophotonics and Nanomaterial based flexible electronics.

\section{Conflicts of Interset}

None.

\section{References}

1. Webb RC, Bonifas AP, Behnaz A, Zhang Y, Yu KJ, et al. (2013) Ultrathin conformal devices for precise and continuous thermal characterization of human skin. Nat Mater 12(10): 938-944.

2. Kim DH, Lu N, Ma R, Kim YS, Kim RH, et al. (2011) Epidermal Electronics. Science 333(6044): 838-843.

3. Daniel JC, Lan Z, Evangelos D, Ivan RM, Katherine MM, et al. (2013) A microchannel neuroprosthesis for bladder control after spinal cord injury in rat. Science Translational Medicine 5(210): 210.

4. Rogers JA, Someya T, Huang Y (2010). Materials and mechanics for stretchable electronics. Science 327(5973): 1603-1607.

5. Kim DH, Ahn JH, Choi WM, Kim HS, Kim TH, et al. (2008) Stretchable and foldable silicon integrated circuits. Science 320(5875): 507-511.

6. Sekitani T, Noguchi Y, Hata K, Fukushima T, Aida T, et al. (2008) A rubberlike stretchable active matrix using elastic conductors. Science 321(5895): 1468-1472. 
7. Sekitani T, Zschieschang U, Klauk H, Someya T (2010) Flexible organic transistors and circuits with extreme bending stability. Nat Mater 9(12): 1015-1022.

8. Kim DK, Lai Y, Diroll BT, Murray CB, Kagan CR (2012) Flexible and low-voltage integrated circuits constructed from high-performance nanocrystal transistors. Nat Commun 3: 1216.

9. Kaltenbrunner M, Sekitani T, Reeder J, Yokota T, Kuribara K, et al (2013) An ultra-lightweight design for imperceptible plastic electronics. Nature 499(7459): 458-463.

10. Xu F, Lu W, Zhu Y (2011) Controlled 3D Buckling of Silicon Nanowires for Stretchable Electronics. ACS Nano 5(1): 672-678.

11. Kang D, Pikhitsa PV, Choi YW, Lee C, Shin SS, et al. (2014) Ultrasensitive mechanical crack-based sensor inspired by the spider sensory system. Nature 516(7530): 222-226.

12. Ryu SY, Xiao J, Park WI, Son KS, Huang YY, et al. (2009) Lateral buckling mechanics in silicon nanowires on elastomeric substrates. Nano Lett 9(9): 3214-3219.

13. Xu F, Wu MY, Safron NS, Roy SS, Jacobberger RM, et al. (2014) Highly stretchable carbon nanotube transistors with ion gel gate dielectrics. Nano Lett 14(2): 682-686.

14. Lee SK, Kim BJ, Jang H, Yoon SC, Lee C, et al. (2011) Stretchable graphene transistors with printed dielectrics and gate electrodes. Nano Lett 11(11): 4642-4646.

15. Novoselov KS, Geim AK, Morozov SV, Jiang D, Zhang Y, et al. (2004). Electric Field Effect in Atomically Thin Carbon Films. Science 306(5696): 666-669.

16. Yao S, Zhu Y (2015) Nanomaterial-enabled stretchable conductors: strategies, materials and devices. Adv Mater 27(9): 1480-1511.

17. Bonaccorso F, Sun Z, Hasan T, Ferrari AC (2010) Graphene photonics and optoelectronics. Nat Photonics 4(9): 611-622.

18. Choi W, Lahiri I, Raghunandan S, Yong SK (2010) Synthesis of Graphene and Its Applications: A Review. Critical Reviews in Solid State and Materials Sciences 35(1): 52-71.

19. Berger C, Song Z, Li X, Wu X, Brown N, et al. (2006) Electronic confinement and coherence in patterned epitaxial graphene. Science 312(5777): 1191-1196.

20. Ohta T, Bostwick A, Seyller T, Horn K, Rotenberg E (2006) Controlling the electronic structure of bilayer graphene. Science 313(5789): 951954.

21. Sutter PW, Flege JI, Sutter EA (2008) Epitaxial graphene on ruthenium. Nat Mater 7(5): 406-411.

22. Eda G, Fanchini G, Chhowalla M (2008) Large-area ultrathin films of reduced graphene oxide as a transparent and flexible electronic material. Nat Nanotechnol 3(5): 270-274.
23. Li X, Zhang G, Bai X, Sun X, Wang X, et al. (2008) Highly conducting graphene sheets and Langmuir-Blodgett films. Nat Nanotechnol 3(9): 538-542.

24. Li X, Cai W, An J, Kim S, Nah J, et al. (2009) Large-area synthesis of high-quality and uniform graphene films on copper foils. Science 324(5932): 1312-1314.

25. Kim KS, Zhao Y, Jang H, Lee SY, Kim JM, et al. (2009) Large-scale pattern growth of graphene films for stretchable transparent electrodes. Nature 457(7230): 706-710.

26. Li X, Zhu Y, Cai W, Borysiak M, Han B, et al. (2009). Transfer of LargeArea Graphene Films for High-Performance Transparent Conductive Electrodes. Nano Lett 9(12): 4359-4363.

27. Bae S, Kim H, Lee Y, Xu X, Park JS, et al. (2010) Roll-to-roll production of 30-inch graphene films for transparent electrodes. Nat Nanotechnol 5(8): $574-578$

28. Wassei JK, Kaner RB (2010) Graphene, a promising transparent conductor. Materials Today 13(3): 52-59.

29. Chen T, Xue Y, Roy AK, Dai L (2014) Transparent and stretchable highperformance supercapacitors based on wrinkled graphene electrodes. ACS Nano 8(1): 1039-1046.

30. Zang J, Ryu S, Pugno N, Wang Q, Tu Q, et al. (2013) Multifunctionality and control of the crumpling and unfolding of large-area graphene. Nat Mater 12(4): 321-325.

31. Chen Z, Ren W, Gao L, Liu B, Pei S, et al. (2011) Three-dimensional flexible and conductive interconnected graphene networks grown by chemical vapour deposition. Nat Mater 10(6): 424-428.

32. Schedin F, Geim AK, Morozov SV, Hill EW, Blake P, et al. (2007) Detection of individual gas molecules adsorbed on graphene. Nat Mater 6(9): 652-655.

33. Tung VC, Chen LM, Allen MJ, Wassei JK, Nelson K, et al. (2009) Lowtemperature solution processing of graphene-carbon nanotube hybrid materials for high-performance transparent conductors. Nano Lett 9(5): 1949-1955.

34. Xu Z, Liu Z, Sun H, Gao C (2013) Highly electrically conductive Agdoped graphene fibers as stretchable conductors. Adv Mater 25(23): 3249-3253.

35. Kholmanov IN, Magnuson CW, Aliev AE, Li H, Zhang B, et al. (2012) Improved electrical conductivity of graphene films integrated with metal nanowires. Nano Lett 12(11): 5679-5683.

36. Lee MS, Lee K, Kim SY, Lee H, Park J, et al. (2013) High-performance, transparent, and stretchable electrodes using graphene-metal nanowire hybrid structures. Nano Lett 13(6): 2814-2821. 